\title{
Use of Colchicine to Treat Severe Constipation in Developmentally Disabled Patients
}

\author{
Paul S. Frame, MD, Peter Dolan, RPA, Rakbi Kobli, and Sbirley W. Eberly, MS
}

Background: Patients with severe developmental disabilities often have concurrent decreased neuromuscular tone of the gastrointestinal tract, which can lead to a weak gag reflex, esophageal reflux, aspiration, and severe intractable constipation. High doses of multiple laxatives are frequently needed to maintain bowel motility in these patients. Colchicine, a natural alkaloid that is primarily used for the treatment of acute attacks of gout, causes an increase in gastrointestinal motility by neurogenic stimulation. The purpose of this study was to determine whether daily colchicine administration can improve bowel function and reduce laxative use in profoundly disabled patients with severe, intractable constipation who currently require large doses of multiple laxatives.

Methods: Twelve developmentally disabled patients who required three or more different laxatives to manage their chronic constipation were selected to participate in a double-blind, crossover study. Eleven patients who completed the study received placebo treatment for 8 weeks and colchicine treatment for 8 weeks. The total number of bowel movements and the total number of laxatives used during each of the two 8-week periods were compared.

Results: Eight of 11 patients experienced an improved bowel pattern while on colchicine compared with placebo, as defined by an increase in total number of bowel movements or a decrease in total number of rectal laxatives used. No clinically important complications were related to use of colchicine.

Conclusions: Colchicine appears to be a valuable adjunct in the management of severe intractable constipation. Larger, long-term studies are needed to confirm these preliminary results. (J Am Board Fam Pract 1998;11:341-6.)

Constipation is the most common digestive complaint in the United States. In most patients it is a minor and easily managed problem. Management of constipation presents a difficult problem, however, in both the elderly and the nonambulatory, severely disabled population. Studies have shown that greater than 50 percent of patients in nursing homes use at least one laxative daily and more than one half of these laxative users took more than 60 doses of laxatives per month. ${ }^{1}$ Death caused by intestinal obstruction secondary to chronic constipation has been reported in mentally and physically handicapped patients. ${ }^{2}$

Submitted, revised, 18 February 1998.

From Tri-County Family Medicine (PSF), Cohocton, the Department of Family Medicine (PSF), and the Department of Biostatistics (SWE), University of Rochester School of Medicine and Dentistry, the Finger Lakes Developmental Disabilities Services Organization (PD), and the University of Rochester School of Medicine and Dentistry (RK), Rochester, NY. Address reprint requests to Paul S. Frame MD, TriCounty Family Medicine, 25 Park Ave, Cohocton, NY 14826.

This project was supported in part by a grant from the Department of Family Medicine at the University of Rochester School of Medicine and Dentistry as part of the summer research fellowship program.
Constipation is frequently defined as having fewer than 3 bowel movements per week. , $^{1,4} \mathrm{Ex}$ cessive straining during bowel movements or a need for regular laxative use can also be included in the definition. The cause of constipation appears to be multifactor: poor dietary intake, lack of adequate fluids, bowel hypotonia, side effects of other medications, and concomitant diseases. Constipation is usually treated by eating a highfiber diet, increasing fluid intake, exercise, and use of laxatives.

Severely retarded and developmentally disabled persons frequently have the most severe problems with constipation, which is often resistant to standard modes of therapy. Chronic constipation occurs in these patients for a number of physiologic reasons: (1) they might have hypotonia and generalized autonomic dysfunction, which greatly reduce bowel motility; (2) their disabilities frequently make them physically inactive; (3) they might have poor oral intake as a result of recurrent aspiration of gastric contents, necessitating placement of gastrostomy feeding tubes; and (4) they might take other medications that aggravate constipation. 
The idea for this study originated while caring for a 34-year-old severely retarded, nonverbal, epileptic man with diffuse hypotonia. The patient had had a gastrostomy feeding tube placed because of recurrent aspiration and episodes of pneumonia. He had intractable constipation, which in spite of taking 36 doses of five different laxatives per week, receiving a high-fiber diet, and maintaining an adequate fluid intake through the feeding tube periodically required multiple $2-\mathrm{L}$ enemas and bisacodyl suppositories to get his bowels to move.

It was noted that whenever the patient was on amoxicillin-clavulanate potassium for pulmonary infections, his bowel function improved dramatically. Diarrhea is a known side effect of this medication. Amoxicillin-clavulanate potassium, however, is not suitable for long-term therapy because of the high risk of developing resistant organisms. The beneficial effect noted with amoxicillin-clavulanate potassium raised the question of whether other, safer agents could be found to improve the patient's constipation. Colchicine, $0.5 \mathrm{mg}$ three times a day, was tried with good results. The patient has had more regular bowel movements, a decreased need for other laxatives, and no need for high-volume enemas. This patient's success led to the hypothesis that colchicine could be a useful treatment for severe refractory constipation.

Colchicine is an alkaloid of Colcbicum autumnale (autumn crocus, meadow saffron). It has been used for pain of articular origin since the 6 th century $\mathrm{AD}$. It is currently used primarily for the treatment of acute attacks of gout, but it can also be used long term to prevent recurrent attacks of gout, pseudogout, familial Mediterranean fever, and progression of amyloidosis. The usual dose is $0.5 \mathrm{mg}$ to 2.0 mg daily. ${ }^{5}$ It is known to enhance gastrointestinal activity by neurogenic stimulation.

A literature search of the use of colchicine to treat constipation found only one citation. In a letter to the fournal of the Royal Society of Medicine in 1984 , Sandyk and Gillman ${ }^{6}$ reported the successful use of colchicine to treat constipation in a patient with Parkinson disease. No studies of the use of colchicine to treat constipation have been conducted. The purpose of this study was to determine whether daily colchicine administration could improve bowel function and reduce laxative use in mentally and physically disabled patients with severe, intractable constipation who currently require large doses of multiple laxatives.

\section{Methods}

Patients were selected from the client population of five state-supported individualized residential alternative homes (IRAs) affiliated with the Finger Lakes Developmental Disabilities Services Organization (DDSO). Each IRA houses approximately 12 mentally and physically disabled patients in a residential home and provides 24 -hour nursing coverage. All of the patients enrolled in this study were incontinent and required diapers and personalized assistance with daily hygiene and feeding. The staff routinely recorded the number and doses of different laxatives used and the number of bowel movements for each patient.

Study participants were selected from patients in these homes who met the following criteria:

1. Twenty-one years of age or older

2. Chronic, severe constipation, defined as requiring three or more different laxative agents on a regular basis for more than 6 months

3. No contraindication to colchicine, specifically liver disease with a serum aspartate aminotransferase (AST) greater than $40 \mathrm{mg} / \mathrm{dL}$ or renal disease with a serum creatinine greater than $1.5 \mathrm{mg} / \mathrm{dL}$

4. Generally stable medical condition. The patients naturally had multiple medical problems, but could not have been in a life-threatening situation during the 6 months before the study or could not have been expected to be in a crisis situation in the 6 months after the study

5. Patients must weigh more than 60 pounds. $\mathrm{Pa}$ tients weighing between 60 and 99 pounds were given colchicine and placebo at a reduced dose twice a day instead of three times a day

6. The patient must have a parent or legal guardian capable of giving informed consent, as approved by the Institutional Review Board, in accordance with the policies of the Finger Lakes DDSO

Twelve patients, 7 women and 5 men, met the criteria and were enrolled in the study. Their ages ranged from 24 to 60 years. All patients had profound mental retardation and epilepsy. Five of the 12 had spastic quadriparesis. One patient was fed through a gastrostomy feeding tube and the others received a high-fiber chopped or pureed diet. All of these patients were taking both oral and rectal laxatives. Oral laxatives were administered on a routine basis, whereas rectal laxatives were given 


\begin{tabular}{|c|c|c|c|c|c|c|c|c|c|c|c|}
\hline Period $^{\star}$ & 1 & 2 & 3 & 4 & 5 & 6 & 7 & 8 & 9 & 10 & 11 \\
\hline Group 1 & \multicolumn{2}{|c|}{ Baseline } & $P t$ & $\mathbf{P}$ & $\mathbf{P}$ & $\mathbf{P}$ & Washout & $\mathrm{C}$ & C & $\mathrm{C}$ & C \\
\hline \multirow[t]{2}{*}{ Group 2} & \multicolumn{2}{|c|}{ Baseline } & $C^{\ddagger}$ & C & C & C & Washout & $\mathbf{P}$ & $\mathbf{P}$ & $\mathbf{P}$ & $\mathbf{P}$ \\
\hline & \multicolumn{2}{|c|}{ PHASE 1} & \multicolumn{4}{|c|}{ PHASE 2} & PHASE 3 & \multicolumn{4}{|c|}{ PHASE 4} \\
\hline
\end{tabular}

Figure 1. Schematic of 22-week study design, comparing colchicine with placebo for laxative effect in treatment of constipation in disabled patients.

*Each period represents 2 weeks.

†P - placebo, 2 or 3 times per day.

${ }^{\ddagger} \mathrm{C}$ - colchicine, $0.6 \mathrm{mg} 2$ or 3 times per day.

on an as-needed basis if there were no bowel movements within a defined period.

The trial was a double-blind, crossover study of the use of colchicine to treat severe constipation in profoundly disabled patients. Figure 1 schematically outlines the study design. The trial lasted 22 weeks and was divided into 4 phases, including 2 8 -week intervention periods. Patients were distributed into two groups. During the 4 weeks of phase 1, the laxative regimen of each patient was optimized to have patients on as few different laxatives and laxative doses as possible without having them experience fewer than 3 bowel movements per week. Patients in group 1 received placebo either orally or by a gastrostomy tube for 8 weeks in phase 2 and colchicine either orally or by gastrostomy tube for 8 weeks in phase 4 . Group 2 patients had the reverse schedule. Phase 3 served as a 2 -week washout period between the two interventions.

The consulting pharmacist prepared the placebos and colchicine and randomly assigned each patient to either group 1 or 2 . The placebos looked similar to the colchicine tablets. For phases 2 and 4 the pharmacist prepared medication vials that listed the name of the patient, the directions for taking the medication, and the dates on which the medication was to be given. Patients heavier than 99 pounds received $0.6 \mathrm{mg}$ of colchicine three times a day and those lighter than 99 pounds received $0.6 \mathrm{mg}$ of colchicine two times a day. The pharmacist had no other role in the care of the patients and did not communicate with the caretakers. The group assignments were not revealed by the pharmacist to the investigators until the end of the study. Thus, both the investigators and the caretakers were unaware of the treatment assignment.

Two of the investigators (PSF and PD) met at the beginning of the study and every 2 weeks to review records of each patient's bowel movements. For ethical reasons, the protocol required that laxatives be adjusted so that patients had at least 3 bowel movements per week but not more than 3 per day. Within these parameters the investigators were free to adjust laxative orders. The study medications (placebo or colchicine) were not adjusted and the investigators were not aware which medication the patient was receiving. All patients were monitored clinically during the study for signs of colchicine toxicity, including diarrhea, vomiting, fever, hair loss, or rash. A complete blood count, 12 -test chemistry panel, creatinine kinase (CK), and single stool sample for occult blood were obtained during phase 1 and after 4 weeks of phases 2 and 4.

The study design and patient consents were reviewed and approved by the Institutional Review Board of the Finger Lakes DDSO. Informed consent was obtained from the parent or legal guardian of each patient selected for the study in accordance with the policies of the Finger Lakes DDSO. In addition, the investigators obtained permission from the patient's attending physician for each patient to participate in the study. To provide uniformity in laxative management, the investigators ordered the patients' laxative medication during the study.

The number of different laxatives used, the number of laxative doses, and the number of bowel movements were recorded for each patient throughout the entire study. The primary comparisons were the total number of bowel movements per 8-week treatment period and the total number of rectal laxatives used per 8-week treatment period. The differences in counts between phases 2 and 4 were calculated for each patient. A one-sample $t$-test was done to determine whether the ag- 
Table 1. Change in Number of Patient's Bowel Movements and Change in Amount of Laxative Use While Taking Colchicine Compared With Placebo.

\begin{tabular}{lrrrrrrrrrrr}
\hline Study Variable & 1 & 2 & 3 & 4 & 5 & 6 & 7 & 8 & 9 & 10 & 11 \\
\hline Bowel movements on colchicine, No & 49 & 54 & 55 & 32 & 30 & 41 & 27 & 35 & 38 & 35 & 24 \\
Bowel movements on placebo, No & 31 & 40 & 44 & 22 & 23 & 34 & 22 & 32 & 45 & 45 & 35 \\
$\begin{array}{l}\text { Change in number of bowel movements } \\
\text { (colchicine minus placebo) }\end{array}$ & 18 & 14 & 11 & 10 & 7 & 7 & 5 & 3 & -7 & -10 & -11 \\
$\begin{array}{l}\text { Change in dosage of rectal laxatives } \\
\text { (colchicine minus placebo) }\end{array}$ & -8 & -5 & -2 & -6 & 1 & -10 & -3 & -4 & 4 & 2 & -5 \\
$\begin{array}{l}\text { Change in dosage of oral laxatives } \\
\text { (colchicine minus placebo) }\end{array}$ & -37 & -155 & -104 & -14 & 0 & 0 & 6 & 0 & 0 & 0 & 48 \\
\hline
\end{tabular}

gregate difference in bowel movements was statistically significant.

\section{Results}

Twelve patients were initially enrolled. One patient was hospitalized during the study for reasons unrelated to the trial. His stool counts were disrupted and his study medication was discontinued for a few days, so this patient was dropped from the trial. As a result, 11 patients constitute the study population.

The results are outlined in Table 1. Eight of 11 patients experienced an increase in number of bowel movements during the 8 -week colchicine treatment period as compared with the 8-week placebo treatment period. We also examined the change in dosage of rectal laxatives. We focused on rectal laxative use in particular because these laxatives, which consist mainly of enemas or suppositories, are more invasive and uncomfortable for the patient compared with oral laxatives. Table 2 describes the effect of colchicine treatment on both bowel movements and rectal laxative use for the 11 patients. Seven of the 8 patients who had an increase in bowel movements on colchicine also required fewer rectal laxatives. One patient (No. 5) experienced an increase in bowel movements and an increase in rectal laxatives while on colchicine. In this case the increase in laxative use was minimal. The patient received one more fleet enema during the 8-week colchicine treatment period compared with the 8 -week placebo treatment period.

Two patients had a decrease in bowel movements while receiving colchicine and also required more rectal laxatives. Colchicine clearly did not improve the bowel pattern of these two patients. One additional patient (No. 11) not only had a de- crease in bowel movements on colchicine, but also had a decrease in rectal laxative use. $\mathrm{Al}$ though at first glance this finding seems rather counterintuitive, during the beginning of phase 2 , while receiving colchicine, this patient was having fewer than 3 bowel movements per week, which prompted us to double his order of senna tablets. Even with this increase he was still severely constipated, which led to our increasing his bisacodyl suppository order during the 7 th week of phase 2 . This increase in bisacodyl suppositories was continued throughout the washout period and phase 4 , thereby resulting in a decrease in rectal laxative use on colchicine compared with placebo. Moreover, that patient No. 11's rectal laxative dosage was increased throughout the placebo period but was increased only during the last 2 weeks of the colchicine period might account for his having more bowel movements with placebo than with colchicine.

An aggregate comparison of the total number of bowel movements while patients were taking colchicine compared with the total number of bowel movements while taking placebo, irrespective of laxative dose, showed that patients experienced on average 4.27 more bowel movements per patient during the 8-week colchicine treatment period. Although this result appears to be clinically significant, it was not statistically significant $(P=$ 0.18 ) because the sample size was small.

Colchicine was well tolerated by all of the patients. No problems relating to the common side effects of colchicine such as nausea, vomiting, diarrhea, or abdominal discomfort were reported by the caretakers. The blood and stool monitoring were all within normal limits with one exception. One patient (No. 11) had an elevated CK level detected at the end of phase 2, later determined to be 
the colchicine treatment period. A repeated CK measurement during the washout period was normal and has continued to be normal on subsequent testing. The patient's CK level was normal at the start of the study.

\section{Discussion}

The primary aim of this study was to determine whether colchicine can improve bowel function in mentally and developmentally disabled patients who currently require large doses of multiple laxatives because of severe, intractable constipation. As shown in Tables 1 and 2, colchicine administration clearly benefited 8 of the 11 patients. Long-term colchicine administration might be an option for these patients.

The average increase in bowel movements per patient during the 8 -week colchicine treatment period was 4.27 . This modest increase was not statistically significant at the $P=0.05$ level $(P<0.18)$. Had we been stricter about not decreasing the dosage of other laxatives unless the patient had more than 3 bowel movements per day, as outlined in the protocol, the increase in number of bowel movements would have been greater. Even though the protocol stated that laxatives did not have to be decreased unless patients experienced more than 3 bowel movements per day, laxatives were often decreased if patients were having more than 1 bowel movement per day. The staff at each IRA had difficulty adjusting to the greater workload that went along with an increase in bowel movements and often complained when patients had more than 1 bowel movement per day. Had we been more stringent with the laxative dosage and kept the patients on a constant dosage of laxatives for both treatment periods, the response to colchicine, as measured by change in number of bowel movements, would probably have been greater.

Limitations of this study include the small sample size and short duration of colchicine administration. The 8-week duration of colchicine administration did not permit study of the maintenance of the laxative effect or the long-term potential side effects of colchicine, such as bone marrow depression, aplastic anemia, alopecia, rashes, myopathy, and peripheral neuritis. The patient who was first given colchicine to treat constipation and who prompted us to design this study, however, has now been receiving colchicine since August 1995 .
Table 2. Change in Bowel Pattern and Rectal Laxative Use on Colchicine Compared With Placebo

\begin{tabular}{llc}
\hline & $\begin{array}{c}\text { Patients With } \\
\text { Decreased Rectal } \\
\text { Laxative Use } \\
\text { on Colchicine }\end{array}$ & $\begin{array}{c}\text { Patients With } \\
\text { Increased or No } \\
\text { Change in Rectal } \\
\text { Laxative Use } \\
\text { on Colchicine }\end{array}$ \\
\hline $\begin{array}{l}\text { Patients having an } \\
\text { increase in bowel } \\
\text { movements } \\
\text { on colchicine }\end{array}$ & 7 & 1 \\
$\begin{array}{l}\text { Patients having a } \\
\text { decrease in bowel } \\
\text { movements } \\
\text { on colchicine }\end{array}$ & 1 & \\
\hline
\end{tabular}

He continues to have a good response and has not had any adverse effects. A second patient, also not part of this study, whom we started on colchicine in 1995 also continues to do well.

Physicians using colchicine to treat chronic constipation will naturally be concerned about possible adverse effects. Acute colchicine toxicity is rare, and when it does occur, it is usually in the setting of renal or hepatic insufficiency or as the result of a large intentional overdose. The initial findings are usually fever, gastrointestinal symptoms, and leukopenia. These symptoms and signs are followed by derangements in multiple organ systems. Survivors undergo a recovery phase characterized by rebound leukocytosis and development of alopecia. ${ }^{7}$ Eighty percent of colchicine is metabolized in the liver primarily by deacetylation and subsequent excretion in the bile. Approximately 20 percent of the parent drug is excreted in the urine. ${ }^{7}$ Patients with severe liver or renal impairment are at increased risk for severe colchicine toxicity unless dosage reductions are made.,8 Colchicine is metabolized by the cytochrome P450 system, so drugs such as erythromycin, cimetidine, and tolbutamide can delay excretion and increase serum levels. ${ }^{7}$

In 1961 Yu and Gutman ${ }^{9}$ reported a study of the efficacy of colchicine prophylaxis in the treatment of gout in 208 patients during a mean period of 5 years and found no evidence of toxicity of the bone marrow, nerves, kidney, or liver. They did not need to discontinue prophylactic use of colchicine because of acquired intolerance to the drug. Thus there are data from other sources that long-term colchicine administration is well tolerated.

The 1 patient in this study who had an elevated 
CK level at the end of the colchicine treatment period might represent a case of colchicine-induced myositis. It is also possible that the abnormally high CK value might simply be a laboratory error, because the patient was asymptomatic throughout the study and the elevated level dropped to within the normal range on subsequent measurements. Until there is more experience using long-term colchicine administration to treat severe constipation, it is certainly prudent for clinicians to monitor patients for clinical and biochemical signs of toxicity. Chronic colchicine administration should be avoided in patients with hepatic or renal insufficiency.

This trial was a short-term study with a small sample size. Nonetheless, the results suggest colchicine might be a valuable treatment option for patients who have severe, intractable constipation. Our intent is not to promote colchicine as a first-line laxative but rather to suggest its potential as an adjunctive form of therapy for difficult-tomanage patients with decreased bowel motility. Colchicine provides an effective alternative for patients with chronic constipation who are resistant to standard modes of therapy. Future studies should include a larger, more diverse patient population and focus on maintenance of the laxative effect as well as surveillance for signs of toxicity. Nursing home patients with severe bowel motility problems might be good candidates for consideration of colchicine therapy.
Colchicine and placebo capsules were prepared by Thomas Quinlan and Quinlan's Pharmacy, Wayland, NY.

\section{References}

1. Harari D, Gurwitz JH, Avorn J, Choodnovskiy I, Minaker KL. Constipation: assessment and management in an institutionalized elderly population. J Am Geriatr Soc 1994;42:947-52.

2. Jancar J, Speller CJ. Fatal intestinal obstruction in the mentally handicapped. J Intellect Disabil Res 1994;38(Pt 4):413-22.

3. Passmore AP, Wilson-Davies K, Stoker C, Scott $M E$. Chronic constipation in long stay elderly patients: a comparison of lactulose and a senna-fibre combination. BMJ 1993;307:769-71.

4. Lamy PP, Krug BH. Review of laxative utilization in a skilled nursing facility. J Am Geriatr Soc 1978;26; 544-9.

5. Insel PA. Analgesic-antipyretic and antiinflammatory agents. In: Gilman AG, Rall TW, Nies AS, Taylor P, editors. Goodman and Gilman's the pharmacological basis of therapeutics. 8th ed. New York: McGraw-Hill, 1990.

6. Sandyk R, Gillman MA. Colchicine ameliorates constipation in Parkinson's disease. J R Soc Med 1984;77:1066.

7. Caraco Y, Putterman C, Rahamimov R, Ben-Chetrit E. Acute colchicine intoxication-possible role of erythromycin administration. J Rheumatol 1992;19: 494-6.

8. Wallace SL, Singer JZ, Duncan GJ, Wigley FM, Kuncl RW. Renal function predicts colchicine toxicity: guidelines for the prophylactic use of colchicine in gout. J Rheumatol 1991;18:264-9.

9. Yu TF, Gutman AB. Efficacy of colchicine prophylaxis in gout. Ann Intern Med 1961;55:179-19. 